\title{
Metformin Loaded Carbopol Gel for lowering the Intra-Abdominal Visceral Fat
}

\author{
Ahmed AH Abdellatif ${ }^{*}$ and Hesham M Tawfeek ${ }^{2}$ \\ ${ }^{1}$ Department of Pharmaceutics and Industrial Pharmacy, Al-Azhar University, Assuit, Egypt \\ ${ }^{2}$ Department of Indsutrial Pharmacy, Assiut University, Assiut, Egypt
}

\begin{abstract}
The aim of this study was to prove the principle of local delivery of metformin hydrochloride, $\mathrm{METHCl}$, to the abdomen area in order to reduce the intra-badominal visceral fat compared to the METHCL oral tablets. Intraabdominal visceral and subcutaneous fat were highly correlated with body weight of obese people. So, this will be beneficial to assist wight loss in those individuals. Furthermore, oral $\mathrm{METHCl}$ tablets $(600 \mathrm{mg})$ might show reduction in weight in diabetic pateints. However, the reqiured high doses is still an issue which put the patients under stress of $\mathrm{METHCl}$ side effects. In this preliminary trial, $\mathrm{METHCl}$ was formulated in the form of local hydrogel using the carbopol 934 within very small drug dose $(6 \mathrm{mg} / \mathrm{g})$. The prepared hydrogel was pharmaceutically evaluated for its $\mathrm{pH}$, viscosity, spreadability, and in vitro release. Furthermore, the abdominal fat lowering activity of $\mathrm{METHCl}$ gel was compared to the oral $\mathrm{METHCl}$ tablets and control (placebo) groups in human volunteers. The study showed that the prepared $\mathrm{METHCl}$ gel significantly ( $<<0.05$, ANOVA/Tukey) decrease the diameter of the abdomen compared to the placebo group and to $50 \%$ of participant taking oral $\mathrm{METHCl}$ tablets after three weeks treatment. In addition, the group taking $\mathrm{METHCl}$ oral tablets failed to decrease the inta-abdominal fat in approximately $50 \%$ of participants. In conclusion, $\mathrm{METHCl}$ hydrogel could be considered a promising novel formulation which can decrease the accumulated fats in the abdomen reigon with small dose comapred to oral $\mathrm{METHCl}$ tablets.
\end{abstract}

Keywords: Metformin hydrochloride; Carbopol gel; Metformin hydrochloride hydrogel; Metformin hydrochloride tablets; Intraabdominal visceral fat

\section{Introduction}

$\mathrm{METHCl}$ is a biguanide antihyperglycemic agent used for treating non-insulin-dependent diabetes mellitus. It is considered to be the first line of choice for the treatment of type 2-diabetes. It could be used also in case of patients with high risk to develop the type 2-diabetes, polycystic ovary syndrome and gestational diabetes [1]. Furthermore, it helps to decrease the LDL, cholesterol and triglycerides levels [2]. Several researchers have been investigated the role of oral $\mathrm{METHCl}$ in weight loss during the treatment of diabetes [3] or in some cases like nonalcohlic fatty liver diseases which associated with obesity [4]. Some researchers pointed out the mechanism of weight loss due to decrease food intake others related this effect was due to changing in circulating steroid hormones and lipid markers [5]. However, in all of these studies a large doses of oral $\mathrm{METHCl}$ was utilised which making the patients under the impact of dangerous side effects like lactic acidosis, lung and liver disorders and gastrointestinal complications.

For management of obesity, life style modification (Diet), physical activity (exercise), feeding behaviour modification, pharmacotherapy and surgery (fat removal) may consedered the ways for obesity treatment [6,7]. Overweight people having a large percentage of fats in the abdomin area are in worse condition compared with equally obese people has fat distributed around their hips and limbs. In addition people with $20 \%$ overweight could be considered a high risk group for different diesease like amenorrhea, cervix cancer, colon, endometrium, gal bladder, prostate, uterus, chronic heart disease and fatty liver $[8,9]$. Drugs like Amphetamine, methamphetamine and phenmetrazine were used in weight loss but not used any more due to their addictive properties and are classified as drug abuse in Egyptain market [10,11]. Some kinds of nanoparticles [12-14], can be used for targeting specific site in the body and can be targeted for fat cells. Phendimetrazine, diethylpropion, phentermine, benzphetamine and mazindol are used for short-term treatments not more than few weeks due to their side effects on lungs and brain $[15,16]$. Other medication like chitosan, a highly effective weight loss agent, which acts as carbohydrate blaster and fat absorption blocker $[17,18]$. Thus, seraching for safe and effective drug delivery method and drugs is considered an issue for weight loss programs.

The aim of our preliminary investigation was to proof the principle of topical delivery of METHCl thus might decreases the accumulated fats in the abdomin area. Thus could be performed through incoporation of METHCl in a sutiable hydrogel base like carbopol followed by administration to the affected area to healthy obease non-diabetic people. Thus, will have a lot of advantages compared to oral $\mathrm{METHCl}$ such as lowering the drug dose, direct application to the affected area and be more convenient to a wide group of patients. The prepared $\mathrm{METHCl}$ hydrogel was examined for its $\mathrm{pH}, \mathrm{METHCl}$ content, spredability, viscosity and in vitro release. Morover, the abdominal fat lowering activity was performed over a group of volunteers and compared with oral $\mathrm{METHCl}$ and placebo groups.

\section{Materials and Method}

\section{Materials}

Metformin hydrochloride is obtained as a gift from CID-Company, Assiut, Egypt. Carbapol 934, sodium hydroxide and methyl Paraben

*Corresponding author: Ahmed $\mathrm{AH}$ Abdellatif, $\mathrm{PhD}$, Department of Pharmaceutics and Industrial pharmacy, Faculty of Pharmacy, Al Azhar University, Assuit 71524 Egypt, Tel: +201016660069; E-mail: ahmed.a.h.abdellatif@azhar.edu.eg

Received February 12, 2016; Accepted April 21, 2016; Published April 28, 2016

Citation: Abdellatif AAH, Tawfeek HM (2016) Metformin Loaded Carbopol Gel for lowering the Intra-Abdominal Visceral Fat. J Bioequiv Availab 8: 149-152. doi:10.4172/jbb.1000286

Copyright: ( 2016 Abdellatif $\mathrm{AAH}$, et al. This is an open-access article distributed under the terms of the Creative Commons Attribution License, which permits unrestricted use, distribution, and reproduction in any medium, provided the original author and source are credited. 
were purchased from Merck Company (Darmstadt, Germany). All other chemicals and reagents were of analytical grades and used as received.

\section{Preparation of METHCl hydrogels and characterisation}

METHCl hydrogel containing 2\% w/v carbapol 934 was prepared by dispersing the carbopol in in distilled water by stirring and heating at $50^{\circ} \mathrm{C}$ until homogenous gel is formed. Sodium hydorxide solution was added to the already formed hydrogel to neutralize the carbopol. Thus was followed by addition of $600 \mathrm{mg}$ METHCl $(6 \mathrm{mg} / \mathrm{g}$ gel $)$ to carbopol hydrogel under stirring at room temperature for $1 \mathrm{~h}$. Any trapped air bubbles has been removed by sonication for $10 \mathrm{~min}$ [1921 ], finally, $0.01 \%$ of methyl paraben was added as a preservative. In addition, control carbopol gel $2 \% \mathrm{w} / \mathrm{v}$ was prepared for comparison. $\mathrm{METHCl}$ hydrogel was stored in amber colored plastic containers at room temperature of ambient conditions and used for further analysis (Figure 1).

The prepared METHCl hydrogel was characterized for their $\mathrm{pH}$, METHCL contents, viscosity and spreadability. The $\mathrm{pH}$ value was obtained using a digital $\mathrm{pH}$ meter (3500 $\mathrm{pH}$ meter, Jenway, UK). The amount of METHCl contained in carbapol gel was determined by dissolving $100 \mathrm{mg}$ of the formulation in $10 \mathrm{ml}$ of methanol followed by filtration. METHCl content was analyzed using UV-VIS spectrophotometer at $\lambda_{\max }$ of $233 \mathrm{~nm}$. The other components of the gel did not interfere with the spectrophotometric analysis of the drug [19]. The viscosity of the prepared formulations was determined at different angular velocities using a stress programmable rheometer (DV.Ultra, RVDV-111 U), Book field, USA). The rpm was adjusted to 1.5 and using spindle No. 96 at $27.0 \pm 0.1^{\circ} \mathrm{C}$. The rheometer was equipped with cone-plate geometry $(4 / 40)$ operating in the oscillation mode. The gel samples were placed onto the bottom plate of the rheometer then the upper plate was then lowered to a gap size of $1000 \mu \mathrm{m}$. Storage (G') and loss modulus (G") were recorded at $27^{\circ} \mathrm{C}$ and $1 \mathrm{~Hz}$ oscillatory frequency as a function of the applied stress [22]. In addition, hydrogel Spreadability in $(\mathrm{g} . \mathrm{cm} / \mathrm{sec})$ was determined by applying weight $(20 \mathrm{~g})$ to glass slides $(6 \mathrm{~cm}$, length) into which formulation was placed. Followed by recording the time in seconds [23] required to separate the slides. Spreadability was then calculated by using the equation:

$$
S=\frac{M \cdot L}{T}
$$

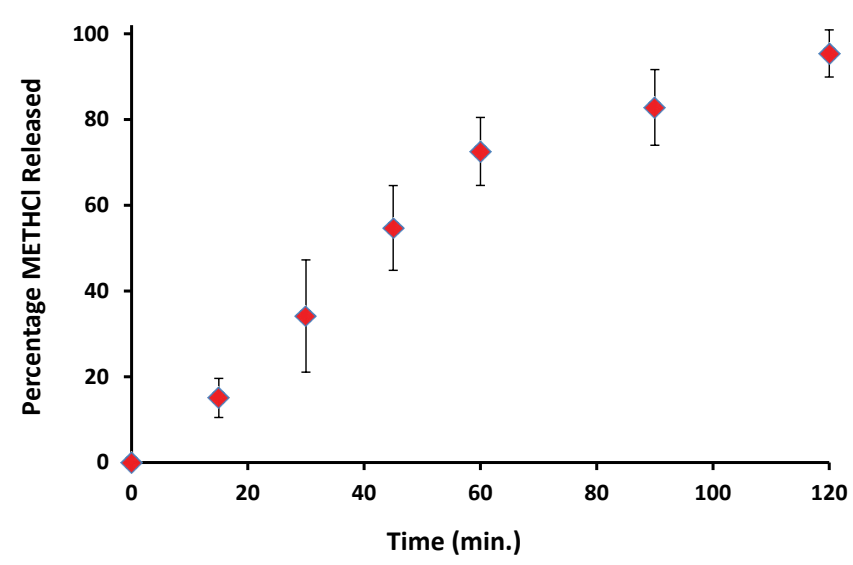

Figure 1: Percentage METHCl released from carbopol hydrogel $2 \% \mathrm{w} / \mathrm{v}$ using a cellophane membrane, data are presented as a mean \pm S.D, $(n=3)$.
Where, S: Spreadability; M: Weight tide to upper slide; L: Length of glass slide; T: Time taken in seconds to separate the slide completely from each other.

\section{In vitro release study}

The in vitro release of METHCl has been performed in apparatus resemble to Franz diffusion cell. Briefly, cellophane membrane was stretched over the end of an open-ended glass tube and made water tight using a rubber band. The tube was immersed vertically in a 100 $\mathrm{mL}$ beaker containing $125 \mathrm{~mL}$ of phosphate buffer ( $\mathrm{pH}$ 7.4) maintained in a thermostatically controlled shaker, 50 stroke/min maintained at $37.0 \pm 1.0^{\circ} \mathrm{C}$ (WiseBath, WSB-45 DAIHAN Scientific Co., Ltd, Korea) [24]. METHCL gel formulation (1.0 g gel equivalent to $6 \mathrm{mg}$ METHCL) was placed into the glass tube. At predetermined time intervals for up to $2 \mathrm{~h}, 5 \mathrm{ml}$ aliquots of the release medium were withdrawn for analysis and were replaced with equal volume of release medium, phosphate buffer, at the same temperature to maintain constant volume. The absorbance of the collected samples was measured spectrophotometer at $\lambda_{\max }$ of $233 \mathrm{~nm}$. The results are reported as the mean values of three release experiments.

\section{Abdominal fat lowering activity}

The preliminary abdominal fat lowering activity of $\mathrm{METHCl}$ gel was performed in three groups of volunteers, each group has 6 participants. The first group taken freshly prepared $\mathrm{METHCl}$ gel, the second group taken plain carbopol gel without drug (placebo group). The first and second groups applied the gel once a day. The third group taken the oral METHCl tablets containing $500 \mathrm{mg}$ drug twice daily. The chosen individuals were healthy non-diabetic within the age of 22-45 and have a fatty abdomen. Volunteers have informed with the nature of the formulation and straight forward to test after obtaining a written informed consent of them. A requisite approval of the Institutional Ethics Committee of Faculty of Medicine, Assiut University, Assiut, Egypt was obtained prior to conduct the study. In this study, the diameter of the abdomen was measured using sagittal abdominal diameter (SAD) which measured the fat masses in the gut region presented in the distance from the small of the back to the upper abdomen [25]. SAD was measured and recorded for three consecuative weeks and compared to both the oral $\mathrm{METHCl}$ and placebo groups.

\section{Statistical analysis}

All statistical analysis was performed using One-way analysis of variance (ANOVA) [26]. Minitab 17 Statistical Software with the Tukey's multiple comparison was employed for comparing the groups with each other from the beginning of experiment and after three weeks treatment. Statistically significant differences were assumed when $\mathrm{p}<0.05$. All values are expressed as their mean \pm standard deviation.

\section{Results and Discussion}

\section{Evaluation of METHCl hydrogel and in vitro release}

The results for $\mathrm{pH}$, drug content, viscosity and spreadability of METHCl hydrogel are presented in Table 1. METHCl hydrogel was found to have a good homogeneity and absences of any lumps when pressed between the thumb and the index finger. Moreover, they were uniform in consistency and free from any appreciable particulate matter upon their microscopic evaluation. In addition, analysis of gel homogeneity and METHCl content after one month storage at room temperature revealed that $\mathrm{METHCl}$ preserve its physical shape, appearance and had a drug content of $98.55 \pm 3.24 \%$. Moreover, the 
Citation: Abdellatif AAH, Tawfeek HM (2016) Metformin Loaded Carbopol Gel for lowering the Intra-Abdominal Visceral Fat. J Bioequiv Availab 8: 149-152. doi:10.4172/jbb. 1000286

$\mathrm{pH}$ value was nearly similar to the skin $\mathrm{pH}$ value which indicated the compatibility of this formulation with the skin [19-21]. The METHCl content in carbopol gel was found to be $98.45 \pm 2.33 \%$ which is considered satisfactory. In addition, METHCL gel showed a higher viscosity and spreadability values as depicted in Table 1 . Higher viscosity made this formulation more suitable for transdermal delivery. Spreadability term is very important to donate the area to which gel readily spreads on application on skin or affected area. On the other hand, higher values of spreadability of the $\mathrm{METHCl}$ gel could indicate that the gel is easily spreadable by small amount of sheer. Regarding the in vitro release, it was found that, $\mathrm{METHCl}$ was efficiently released from carbopol gel, approximately $95.40 \pm 5.49 \%$ was released after 2 $\mathrm{h}$ in Phosphate buffered saline of $\mathrm{pH}$ 6.8. This higher in vitro release possibly attributed to the high water solubility of the drug hence better absorption.

\section{Abdominal fat lowering activity}

The preliminary abdominal fat lowering activity study has shown that the group receiving $\mathrm{METHCl}$ gel has a significant reduction $(\mathrm{p}<0.05$, ANOVA/Tukey) in the diameter of the abdomen after 3 weeks of treatment compared to the beginning of the study ( 0 time) and the placebo group as shown in Table 2 . All volunteers were male; the body weights ranged from $95 \pm 6 \mathrm{~kg}$, and body mass index (BMI) of $30 \pm 5$. All volunteers were provided it with the same calorie as to prove the effect of METHCL gel. However, the placebo group does not show any further reduction in the diameter of the abdomen after 3 weeks treatment. On the other hand, the group receiving oral $\mathrm{METHCl}$ showed a significant reduction in the diameter of the abdomen in half of participants compared to the 0 time and placebo group after 3 weeks. Furthermore, comparing the group receiving $\mathrm{METHCl}$ hydrogel with oral $\mathrm{METHCl}$ a significant difference $(p<0.05$, ANOVA/Tukey) was found between 5 participants in the first group and the third group. Both oral and gel preparations of $\mathrm{METHCl}$ has a pronounced effect on decreasing the accumulated lipids and fats on the abdomen region however, oral $\mathrm{METHCl}$ failed to demonstrate such effect in approximately $50 \%$ of participants compared to the first group which took METHCl gel. Furthermore, the high doses ( $1000 \mathrm{mg}$ daily) were still an issue regarding the dangerous side effects. Moreover, $\mathrm{METHCl}$ gel was successfully decreased the abdomen fats in $100 \%$ of participants with a lower dose ( $6 \mathrm{mg} / \mathrm{g}$ gel, once daily). In addition, we cannot neglect the nature of the formulation which is easy and more comfortable for application for a wide range of population in comparison to the large $\mathrm{METHCl}$ tablets which is not preferred in some people. We can conclude the superior activity of $\mathrm{METHCl}$ hydrogel formulation compared to oral $\mathrm{METHCl}$ in lowering the abdomen fats and lipids within a lower dose and easy of application. Further investigations are in progress to demonstrate the mechanism of action in lowering the abdomen fat in both cellular and animal models. As well as optimizing the pharmaceutical formulation using different gel bases and other additives.

\section{Conclusion}

METHCl was successfully formulated in the form of carbopol hydrogel $2 \% \mathrm{w} / \mathrm{v}$. The prepared hydrogel showed an acceptable pharmaceutical behaviours regarding, $\mathrm{pH}$, drug content, viscosity and spreadability values which demonstrated a more comfortable application to the volunteers. Moreover, $\mathrm{METHCl}$ hydrogel was stable

\begin{tabular}{|c|c|c|c|c|}
\hline Formulation & pH & Drug Content (\%) & Viscosity (cPs) & Spreadability (g.cm/sec) \\
\hline METHCl hydrogel & $6.7 \pm 0.2$ & $98.45 \pm 2.33$ & $267 \times 10^{3} \pm 30.5$ & $5.7 \pm 0.4$ \\
\hline
\end{tabular}

Table 1: The $\mathrm{pH}, \mathrm{METHCl}$ content, viscosity and Spreadability of $\mathrm{METHCl}$ carbopol gel. Data are presented as a mean \pm S.D, $(n=3)$.

\begin{tabular}{|c|c|c|c|c|}
\hline \multirow{2}{*}{ Volunteers } & \multicolumn{4}{|c|}{ Treatments with METHCI } \\
\hline & 0 time & After one week & After two weeks & After three weeks \\
\hline \multicolumn{5}{|c|}{ Group received METHCI hydrogel (applied one time daily/each $1 \mathrm{~g}$ gel contain 6 mg drug) } \\
\hline Participant 1 & $126.0 \pm 0.8$ & $113.9 \pm 0.3$ & $111.1 \pm 2.0$ & $103.3 \pm 0.6^{*, \star *}$ \\
\hline Participant 2 & $120.6 \pm 1.4$ & $118.4 \pm 0.5$ & $112.3 \pm 2.7$ & $108.9 \pm 1.9^{*}$ \\
\hline Participant 3 & $129.5 \pm 1.0$ & $123.3 \pm 1.3$ & $99.8 \pm 0.5$ & $97.9 \pm 1.2^{*, * *}$ \\
\hline Participant 4 & $125.4 \pm 1.7$ & $126.6 \pm 0.3$ & $112.5 \pm 1.2$ & $105.2 \pm 0.4^{*}$ \\
\hline Participant 5 & $124.1 \pm 1.6$ & $120.2 \pm 1.2$ & $111.9 \pm 1.1$ & $110.0 \pm 1.5^{*}$ \\
\hline Participant 6 & $120.9 \pm 2.7$ & $112.1 \pm 1.6$ & $98.6 \pm 0.9$ & $97.6 \pm 1.0^{\star, \star \star}$ \\
\hline \multicolumn{5}{|c|}{ Group received Plain Gel (placebo) } \\
\hline Participant 1 & $121.0 \pm 0.1$ & $125.0 \pm 0.12$ & $122.0 \pm 0.22$ & $129.0 \pm 0.77$ \\
\hline Participant 2 & $123.6 \pm 0.9$ & $121.6 \pm 0.88$ & $124.8 \pm 0.77$ & $127.6 \pm 0.98$ \\
\hline Participant 3 & $125.5 \pm 0.4$ & $126.5 \pm 0.77$ & $133.5 \pm 0.5$ & $125 \pm 0.85$ \\
\hline Participant 4 & $123.4 \pm 0.7$ & $121.4 \pm 0.9$ & $126.1 \pm 0.95$ & $118 \pm 0.99$ \\
\hline Participant 5 & $120.1 \pm 1.1$ & $120.1 \pm 1.6$ & $127.1 \pm 1.6$ & $125 \pm 0.9$ \\
\hline Participant 6 & $121.9 \pm 1.2$ & $128.9 \pm 1.1$ & $128.1 \pm 1.88$ & $136 \pm 3.1$ \\
\hline \multicolumn{5}{|c|}{ Group received oral METHCI $(500 \mathrm{mg} /$ tablet $) /$ twice daily } \\
\hline Participant 1 & $121.0 \pm 0.1$ & $118.6 \pm 1.1$ & $117.0 \pm 0.9$ & $116.1 \pm 0.19$ \\
\hline Participant 2 & $123.6 \pm 0.9$ & $119.1 \pm 1.5$ & $116.9 \pm 1.19$ & $114.4 \pm 0.7^{*}$ \\
\hline Participant 3 & $125.5 \pm 0.4$ & $119.9 \pm 0.8$ & $117 \pm 0.1$ & $112 \pm 0.5^{\star}$ \\
\hline Participant 4 & $123.4 \pm 0.7$ & $121 \pm 1$ & $114.96 \pm 0.5$ & $114.1 \pm 0.2^{*}$ \\
\hline Participant 5 & $120.1 \pm 1.1$ & $120 \pm 1.7$ & $119 \pm 1.16$ & $118 \pm 0.1$ \\
\hline Participant 6 & $121.9 \pm 1.2$ & $129.6 \pm 1.3$ & $122.1 \pm 1.6$ & $119.2 \pm 1.5$ \\
\hline
\end{tabular}

*: Significant difference $(p<0.05$, ANOVA/Tukey) compared to zero time and placebo group (week 3$)$

**: Significant difference ( $p<0.05$, ANOVA/Tukey) compared to group receiving oral METHCI tablets.

Data are presented as a mean \pm S.D. ( $n=6$ for each group).

Table 2: Comparison between $\mathrm{METHCl}$ hydrogel, plain placebo gel (control group) and oral $\mathrm{METHCl}$ in lowering the diameter of the abdomen area in different volunteers 
Citation: Abdellatif AAH, Tawfeek HM (2016) Metformin Loaded Carbopol Gel for lowering the Intra-Abdominal Visceral Fat. J Bioequiv Availab 8: 149-152. doi:10.4172/jbb.1000286

after one month storage at room conditions. METHCl hydrogel $(6 \mathrm{mg} / \mathrm{g}$ gel) showed a significant reduction $(\mathrm{p}<0.05$; ANOVA/Tukey) in the diameter of the abdomen area after three weeks treatment compared to initial time (0 time), placebo control group and to $50 \%$ of the third group receiving oral $\mathrm{METHCl}$ (1000 mg/daily).

\section{References}

1. Fung CS, Wan EY, Wong CK, Jiao F, Chan AK (2015) Effect of metformin monotherapy on cardiovascular diseases and mortality: a retrospective cohort study on Chinese type 2 diabetes mellitus patients. Cardiovasc Diabetol 14 : 137

2. Bolen S, Feldman L, Vassy J, Wilson L, Yeh HC, et al. (2007) Systematic review: comparative effectiveness and safety of oral medications for type 2 diabetes mellitus. Ann Intern Med 147: 386-399.

3. Lee A, Morley JE (1998) Metformin decreases food consumption and induces weight loss in subjects with obesity with type II non-insulin-dependent diabetes. Obes Res 6: 47-53.

4. Krakoff J, Clark JM, Crandall JP, Wilson C, Molitch ME, et al. (2010) Effects of metformin and weight loss on serum alanine aminotransferase activity in the diabetes prevention program. Obesity (Silver Spring) 18: 1762-1767.

5. Harborne LR, Sattar N, Norman JE, Fleming R (2005) Metformin and weight loss in obese women with polycystic ovary syndrome: comparison of doses. $J$ Clin Endocrinol Metab 90: 4593-4598.

6. Behary P, Cegla J, Tan TM, Bloom SR (2015) Obesity: Lifestyle management bariatric surgery, drugs, and the therapeutic exploitation of gut hormones. Postgrad Med 127: 494-502.

7. Nordstrand N, Hertel JK, Hofso D, Sandbu R, Saltvedt E, et al. A controlled clinical trial of the effect of gastric bypass surgery and intensive lifestyle intervention on nocturnal hypertension and the circadian blood pressure rhythm in patients with morbid obesity. Surgery 151: 674-680.

8. Miyawaki T, Azuma N, Fukunaga Y, Kobayashi N (2011) Abdomen specific bioelectrical impedance analysis (BIA) methods for evaluation of visceral fat accumulation. Nihon Rinsho 69 Suppl 1: 470-472.

9. Reddy MP, Sangster G, Heldmann MG, Lilien DL (2006) Fat necrosis in the abdomen after surgery close to the pancreas: Potential false-positive on positron emission tomography. Clin Nucl Med 31: 499-500.

10. Fazio RM, Chen O, Eldarawy W (2015) Superior Mesenteric Artery Syndrome Associated with Rapid Weight Loss Attributed to Amphetamine Abuse. Case Rep Gastrointest Med 2015: 817249.

11. Harris SC, Ivy AC, Searle LM (1947) The mechanism of amphetamine-induced loss of weight; a consideration of the theory of hunger and appetite. J Am Med Assoc 134: 1468-1475.
12. Abdellatif AAH (2015) Targeting of somatostatin receptors using quantum dots nanoparticles decorated with Octreotide. J Nanomed Nanotechnol S6: 005.

13. Abdellatif AA, El Rasoul SA, Osman S (2015) Gold nanoparticles decorated with octreotide for somatostatin receptors targeting. Int J Pharm Sci Res 7 : $14-20$

14. Abdellatif AA, Zayed G, El-Bakry A, Zaky A, Saleem IY, et al. (2016) Nove gold nanoparticles coated with somatostatin as a potential delivery system for targeting somatostatin receptors. Drug Dev Ind Pharm.

15. Le riche WH, Van Belle G (1962) Study of phendimetrazine bitartrate as an appetite suppressant in relation to dosage, weight loss and side effects. Can Med Assoc J 87: 29-31.

16. Johnson WG, Hughes JR (1979) Mazindol: its efficacy and mode of action in generating weight loss. Addict Behav 4: 237-244.

17. Mhurchu CN, Dunshea-Mooij C, Bennett D, Rodgers A (2005) Effect of chitosan on weight loss in overweight and obese individuals: a systematic review of randomized controlled trials. Obes Rev 6: 35-42.

18. Shields KM, Smock N, McQueen CE, Bryant PJ (2003) Chitosan for weight loss and cholesterol management. Am J Health Syst Pharm 60: 1310-1312, 1315-1316.

19. Abdellatif AA, Tawfeek HM (2015) Transfersomal Nanoparticles for Enhanced Transdermal Delivery of Clindamycin. AAPS PharmSciTech.

20. Abdellatif AA, Abou-Taleb HA (2015) Optimization of nano-emulsion formulations for certain emollient effect. WJPPS 14: 1314-1328.

21. Abdellatif AAH, Abou-Taleb HA (2016) Transfersomal Nanoparticles of Keratolytic and Antibacterial Agents for Enhanced Transdermal Delivery. J Nano Adv Mat 4: 19-23.

22. Osman SK, Soliman GM, EI Rasoul SA (2015) Physically cross-linked hydrogels of $\hat{I}^{2}$-cyclodextrin polymer and poly(ethylene glycol)-cholesterol as delivery systems for macromolecules and small drug molecules. Curr Drug Deliv 12: 415-424.

23. Liberman, Rieger MM, Banker GS (1989) Pharmaceutical Dosage Form, Disperse Systems. Journal of Pharmaceutical Sciences 79: 856.

24. El Hamd MA, Abdellatif AAH, Derayea SM, Abdelmageed OH, Askal HF (2015) Spectrophotometric Determination of Nifedipine and Nicardipine in their Pharmaceutical Preparations. Ind Chem Open Access 1:103.

25. Iribarren C, Darbinian JA, Lo JC, Fireman BH, Go AS (2006) Value of the sagittal abdominal diameter in coronary heart disease risk assessment: cohort study in a large, multiethnic population. Am J Epidemiol 164: 1150-1159.

26. Abdellatif AAH, El Hamd MA, Saleh KI (2016) A Formulation, Optimization and Evaluation of Controlled Released Alginate Beads Loaded-Flurbiprofen. $J$ Nanomed Nanotechnol 7: 357. 